\title{
Model Completion via Deformation Cloning Based on an Explicit Global Deformation Model ${ }^{\star}$
}

\author{
Qiong $\operatorname{Han}^{1}$, Stephen E. Strup ${ }^{2}$, Melody C. Carswell ${ }^{1}$, Duncan Clarke ${ }^{1}$, \\ and Williams B. Seales ${ }^{1}$ \\ 1 Center for Visualization and Virtual Environments, University of Kentucky, USA \\ 2 Division of Urology, Department of Surgery, University of Kentucky, USA \\ qhan@engr.uky.edu
}

\begin{abstract}
Our main focus is the registration and visualization of a pre-built 3D model from preoperative images to the camera view of a minimally invasive surgery (MIS). Accurate estimation of soft-tissue deformations is key to the success of such a registration. This paper proposes an explicit statistical model to represent global non-rigid deformations. The deformation model built from a reference object is cloned to a target object to guide the registration of the pre-built model, which completes the deformed target object when only a part of the object is naturally visible in the camera view. The registered target model is then used to estimate deformations of its substructures. Our method requires a small number of landmarks to be reconstructed from the camera view. The registration is driven by a small set of parameters, making it suitable for real-time visualization.
\end{abstract}

\section{Introduction}

The distinct advantage of minimally invasive surgery (MIS) is that it induces less trauma to patients. Preoperative images reveal important substructures of target objects, which are unfortunately not visible under a laparoscopic camera view. Incorporating preoperative images into MIS is thus focused by many researchers. Among different approaches, reconstructing 3D points from a camera video sequence and registering a pre-built $3 \mathrm{D}$ model to the reconstructed $3 \mathrm{D}$ points has the strength of converting the $3 \mathrm{D}$-to- $2 \mathrm{D}$ registration to a $3 \mathrm{D}$-to- $3 \mathrm{D}$ registration.

Devernay et al. proposed a 5 step method for augmented reality of cardiac MIS [1. 2] uses stereo images to reconstruct dense depth cues of surgical scenes. [3] fused stereo depth cues with monocular depth cues based on surface shading. Stereo based methods in general require repeatable tracking of a large number of feature points in order to reconstruct a dense set of surface points. Structure from motion (SFM) method is also adapted to MIS, and Hu et al. used a Competitive

\footnotetext{
* We thank the Medical Image Display \& Analysis Group (MIDAG) at UNC-Chapel Hill for providing the kidney data and source codes. This work was done with support from U.S. Army grant W81XWH-06-1-0761.
} 
Evolutionary Agent-based (CEA) method to deal with the missing data problem in SFM 4 .

Statistical models of deformations and motions have also been proposed. In [5], a statistical deformation model is built from simulated finite element model (FEM) deformations of the prostate. However, proper tissue property parameters are difficult to determine for FEM simulations. [6] explicitly included material properties in their FEM simulations and built a statistical motion model to guide the deformation estimation of the prostate. [7] proposed an explicit 1D motion model to represent and compensate the motion of the mitral valve annulus.

Our driving clinical applications are laparoscopic cryoablation and laparoscopic partial nephrectomy on small renal tumors. 3D visualization of a kidney and its tumor is expected to increase the positioning accuracy of the tumor. Furthermore, surgical plans based on the same preoperative scans, from which the 3D model is built, can be visualized in real time to improve the precision of needle insertion for cryoablation or of incision site and depth for partial nephrectomy. The challenge is that there are always non-rigid intra-object deformations between the kidney in the CT scans and the kidney during an MIS. This paper proposes an explicit global deformation model, which is statistically built from a reference object and its deformed shapes. Furthermore, training data to learn a deformation model is sometimes difficult to acquire. Therefore, we propose to clone a learned deformation model to a new target object to guide the registration of the target object into the camera view, based on a small number of landmarks reconstructed from a camera video sequence.

Next section details our proposed method by its main steps. Section 3 describes the evaluation of the proposed method and shows the results. Section 4 concludes the paper with discussions.

\section{Method}

Our method takes a 5 -step process shown as follows and detailed in the following subsections.

1. Build a statistical deformation model from a reference object and its deformed shapes;

2. Build a 3D model of a target object from the pre-operative computedtomography $(\mathrm{CT})$ scans;

3. Capture a video sequence of the exposed target object with a calibrated laparoscopic camera, and reconstruct 3D landmarks of the target object using the SFM method;

4. Clone the statistical deformation model to the target object model to register the target model to the reconstructed 3D landmarks;

5. Apply the deformation of the registered target object to its substructures.

\subsection{A Statistical Deformation Model}

The discrete m-rep $[8]$ is chosen as the shape model because of its unique property of modeling and parameterizing both the surface and the interior volume of 
an object. A discrete m-rep $\mathbf{M}$ consists of a quad-mesh of $n_{M}$ medial atoms $\left\{\mathbf{m}_{i}, i=1,2, \ldots, n_{M}\right\}$. Each internal atom $\mathbf{m}_{i}$ has a hub position $\mathbf{p}_{i}$, two spokes $\mathbf{S}_{i}^{+1,-1}$ with a radius $r_{i}$ and direction $\mathbf{U}_{i}^{+1,-1}$. Atoms at the edge of the quadmesh are treated differently. For simplicity, all medial atoms are considered the same in this paper.

To learn a statistical deformation model based on m-reps, a series of deformed shapes of a reference object are captured either by a set of CT scans or by a series 3D reconstructed meshes. This paper uses the latter. An m-rep is fitted to each mesh to form a training set of m-reps. Principal geodesic analysis (PGA) 9 is applied to the training set of m-reps to form a statistical deformation model, given as a Frechét mean $\overline{\mathbf{M}}$, the first $n_{P G A}$ principal geodesic directions $\mathbf{v}_{j}, j=$ $1,2, \ldots, n_{P G A}$, representing more than $95 \%$ of the total deformation variations, and the corresponding variances $\lambda_{j}$ of the principal geodesic directions.

Now given a set of principal geodesic components $c_{j} \in \mathbb{R}, j=1,2, \ldots, n_{P G A}$, a deformed reference object $\mathbf{M}_{P G A}$ can be reconstructed from $\overline{\mathbf{M}}$ and a tangent vector $\sum_{j=1}^{n_{P G A}} c_{j} \mathbf{v}_{j}$ via the exponential map [9]. The deformation between $\mathbf{M}_{P G A}$ and $\overline{\mathbf{M}}$ can be represented by the residue between the two m-reps [10, which is defined as the set of residues between all corresponding atom pairs $\left(\mathbf{m}_{P G A, i}, \overline{\mathbf{m}}_{i}\right)$.

Each medial atom is an element of a Riemannian symmetric space $\mathcal{G}=\mathbb{R}^{3} \times$ $\mathbb{R}^{+} \times \mathcal{S}^{2} \times \mathcal{S}^{2}$. The following operator defines the difference between a pair of atoms $\left(\mathbf{m}_{P G A, i}, \overline{\mathbf{m}}_{i}\right)$ :

$$
\mathbf{m}_{P G A, i} \ominus \overline{\mathbf{m}}_{i}=\left(\mathbf{p}_{P G A, i}-\overline{\mathbf{p}}_{i}, \frac{r_{P G A, i}}{r_{i}}, \mathbf{R}_{\mathbf{S}_{P G A, i}^{+1}}\left(\overline{\mathbf{S}}_{i}^{+1}\right), \mathbf{R}_{\mathbf{S}_{P G A, i}^{-1}}\left(\overline{\mathbf{S}}_{i}^{-1}\right)\right)
$$

where for any $\mathbf{w}=\left(w_{1}, w_{2}, w_{3}\right) \in \mathcal{S}^{2}, \mathbf{R}_{\mathbf{w}} \in \mathcal{S O}(3)$ is the rotation around the axis passing the origin $(0,0,0)$ and $\left(w_{2},-w_{1}, 0\right)$ with the rotation angle being the geodesic distance between a chosen point $\mathbf{p}_{0}=(0,0,1)$ and $\mathbf{w}$ on the unit sphere. Let $\Delta \mathbf{m}_{i}=\mathbf{m}_{P G A, i} \ominus \overline{\mathbf{m}}_{i} . \Delta \mathbf{m}_{i}$ is also an element of $\mathcal{G}$, and it is called the residue of $\mathbf{m}_{P G A, i}$ to $\overline{\mathbf{m}}_{i}$, which records the deformation of $\mathbf{m}_{P G A, i}$ relative to $\overline{\mathbf{m}}_{i}$ 's coordinates.

The residue, i.e., the deformation, between a pair of atoms can then be cloned to a new atom via an operator $\oplus$ :

$$
\mathbf{m}_{i} \oplus \Delta \mathbf{m}_{i}=\left(\mathbf{p}_{i}+\Delta \mathbf{p}_{i}, r_{i} \dot{\Delta r_{i}}, \mathbf{R}_{\mathbf{S}_{i}^{+1}}^{-1}\left(\Delta \mathbf{S}_{i}^{+1}\right), \mathbf{R}_{\mathbf{S}_{i}^{-1}}^{-1}\left(\Delta \mathbf{S}_{i}^{-1}\right)\right)
$$

where $\mathbf{R}_{\mathbf{w}}^{-1} \in \mathcal{S O}(3)$ is the inverse rotation of $\mathbf{R}_{\mathbf{w}}$.

Based on operators $\ominus$ and $\oplus$, the residue $\Delta \mathbf{M}$ between two m-reps $\mathbf{M}_{P G A}$ and $\overline{\mathbf{M}}$ and the deformation cloning of $\Delta \mathbf{M}$ to a target m-rep $\mathbf{M}_{t}$ are defined as follows:

$$
\begin{aligned}
\Delta \mathbf{M} & =\mathbf{M}_{P G A} \ominus \overline{\mathbf{M}}=\left\{\Delta \mathbf{m}_{i}, i=1,2, \ldots, n_{M}\right\} \\
\mathbf{M}_{\text {deformed,t }} & =\mathbf{M}_{t} \oplus \Delta \mathbf{M}=\left\{\mathbf{m}_{t, i} \oplus \Delta \mathbf{m}_{i}, i=1,2, \ldots, n_{M}\right\} .
\end{aligned}
$$

$\Delta \mathrm{M}$ is the explicit statistical deformation model learned from the reference object, which is a function of the principal geodesic components $\left\{c_{j}\right\}$. 


\subsection{A Pre-built 3D Model for the Target Object}

The 3D m-rep model $\mathbf{M}_{t}$ is built from a manual segmentation of pre-operative CT scans of the target object by experts, and an m-rep is fitted into the segmentation using the binary fitting method described in [11. An automatic segmentation tool will be highly desirable for this step.

\subsection{Reconstruction of 3D Landmarks}

Using the structure from motion (SFM) method, a dense set of object surface points $\mathbf{L}_{\text {all }}=\left\{\mathbf{l}_{k}, k=1,2, \ldots, N_{L_{\text {all }}}\right\}$ are reconstructed from a laparoscopic video sequence of the target object. A small subset of $\mathbf{L}_{\text {all }}$ are identified as a set of 6 to 9 anatomical landmarks $\mathbf{L}=\left\{\mathbf{l}_{k}, k=1,2, \ldots, n_{L}\right\}$. At the same time, an initial correspondence is established between the set of landmarks $\mathbf{L}$ and a set of surface points on the m-rep $\mathbf{M}_{t}$. This correspondence will, however, be automatically updated in the registration step whenever necessary, via the iterative closest point (ICP) method [12].

In order to get a robust reconstruction of the landmarks, fiducial markers can be used because of the small size of the landmark set. Although this step is not the main focus of this paper, the accuracy of the $3 \mathrm{D}$ reconstruction is crucial to the consequent steps. The effect of reconstruction errors on the registration step are evaluated in section 3 ,

\subsection{Model Registration via Deformation Cloning}

By cloning $\Delta \mathbf{M}$ to a target m-rep $\mathbf{M}_{t}$, we transfer the deformation learned from the reference object to the target object. As a result, we have a specific deformation model for the target object. An alignment step is required to properly clone a deformation to the target object. The alignment is described first, followed by a full description of the registration step.

Alignment step: in order to properly apply a deformation residue $\Delta \mathbf{M}\left(\left\{c_{j}\right\}\right)$ to $\mathbf{M}_{t}, \mathbf{M}_{t}$ must be aligned to the mean reference object $\overline{\mathbf{M}}$ via a similarity transformation $\mathbf{T}_{\text {sim }}=\left\{\mathbf{p}_{\text {sim }} \in \mathbb{R}^{3}, r_{\text {sim }} \in \mathbb{R}^{+}, \mathbf{R}_{\text {sim }} \in \mathcal{S O}(3)\right\}: \mathbf{T}_{\text {sim }}=$ $\arg \min _{\mathbf{T}} d i s_{\text {geodesic }}^{2}\left(\mathbf{T}\left(\mathbf{M}_{t}\right), \overline{\mathbf{M}}\right)$, where dis geodesic $\left(\mathbf{M}_{1}, \mathbf{M}_{2}\right)$ is the squared geodesic distance between two m-reps $\mathbf{M}_{1}$ and $\mathbf{M}_{2}[9$.

Let $\mathbf{M}_{t}^{\text {aligned }}=\mathbf{T}_{\text {sim }}\left(\mathbf{M}_{t}\right)$. A deformed target object with cloned deformation $\Delta \mathbf{M}$ is defined as $\mathbf{M}_{\text {deformed, } t}=\mathbf{M}_{t}^{\text {aligned }} \oplus \frac{\Delta \mathbf{M}}{r_{\text {sim }}}$, where $r_{\text {sim }}$ is the scaling factor in $\mathbf{T}_{\text {sim }}$, and where $\frac{\Delta \mathbf{M}}{r_{s i m}}=\left\{\frac{\Delta \mathbf{m}_{i}}{r_{s i m}}\right\} \cdot \frac{\Delta \mathbf{m}_{i}}{r_{s i m}}$ means each $\Delta \mathbf{p}_{i} \in \mathbf{m}_{i}$ is replaced by $\frac{\Delta \mathbf{p}_{i}}{r_{s i m}}$ because the translation component in an m-rep atom deformation is scaledependent, but the scaling and rotational components are scale-independent.

$\mathbf{M}_{\text {deformed,t }}$ is then registered (fitted) to the set of reconstructed landmarks $\mathbf{L}=\left\{\mathbf{l}_{k} k=1,2, \ldots, n_{L}\right\}$. For each $\mathbf{l}_{k}$, there is a corresponding surface point $\mathbf{f}_{k}$ on the implied surface of $\mathbf{M}_{t}$. The fitting is implemented by minimizing an objective function:

$$
\mathbf{M}_{t}^{\prime}=\arg \min _{\mathbf{T}_{\text {rigid }}, \mathbf{M}_{\text {deformed }, t}} F\left(\mathbf{T}_{\text {rigid }}\left(\mathbf{M}_{\text {deformed }, t}\left(\left\{c_{j}, j=1,2, \ldots, n_{P G A}\right\}\right)\right)\right)
$$


where $F\left(\mathbf{M}_{t}^{\prime}\right)$ has three components as $F\left(\mathbf{M}_{t}^{\prime}\right)=t_{1} F_{f i t}\left(\mathbf{M}_{t}^{\prime}\right)+t_{2} F_{\text {maha }}\left(\mathbf{M}_{t}^{\prime}\right)+$ $\left(1-t_{1}-t_{2}\right) F_{\text {leg }}\left(\mathbf{M}_{t}^{\prime}\right)$, with $t_{1}, t_{2}$, and $t_{1}+t_{2} \in(0,1)$ as two tuning parameters: $F_{f i t}=\Sigma_{k=1}^{n_{L}}\left(\frac{\operatorname{dis}\left(\mathbf{f}_{k}\left(\mathbf{M}_{t}^{\prime}\right), \mathbf{l}_{k}\right)}{r_{\text {mean }}}\right)^{2}$ measures the fitting quality of the model to the set of landmarks by the Euclidean distance function dis and the geometric mean of the radii of all medial atoms $r_{\text {mean }} ; F_{\text {maha }}=\sum_{i=1}^{n_{P G A}}\left(\frac{c_{j}}{\lambda_{j}}\right)^{2}$ is the squared Mahalanobis distance between the current m-rep $\mathbf{M}_{t}^{\prime}$ and the m-rep $\mathbf{M}_{t}^{\text {aligned }}$ without deformations, penalizing big deformations of $\mathbf{M}_{t}^{\prime} ; F_{l e g}=\Sigma_{i=1} n_{M} f_{l e g}\left(\mathbf{m}_{t, i}^{\prime}\right)$, where $\mathbf{m}_{t, i}^{\prime}$ is a medial atom in $\mathbf{M}_{t}^{\prime}$, and where $f_{\text {leg }}$ is the illegality penalty term defined by equation (12) in 11. This component penalizes shape illegalities, such as creasing or folding.

The overall algorithm is shown as follows:

1. Initialize $\left\{c_{j}\right\}$ to $\{0\}$, and calculate an initial alignment $\mathbf{T}_{\text {rigid }}$ to minimize $F\left(\mathbf{M}_{t}^{\text {aligned }}\right)$;

2. Optimize $F\left(\mathbf{M}_{t}^{\prime}\right)$ over $\left\{c_{j}\right\}$ and $\mathbf{T}_{\text {rigid }}$ via the conjugate gradient method until the objective function converges. Because of the compactness of the deformation model, $n_{P G A}$ is usually smaller than 5 , and the optimization usually converges within 30-40 sub-steps;

3. If $F_{f i t}\left(\mathbf{M}_{t}^{\prime}\right)$ is bigger than an empirically set threshold $\varepsilon$, an iteration of ICP is used to re-establish the correspondence between $\mathbf{M}_{t}^{\prime}$ and the landmark set L, and go back to step 2 .

Step 3 is often not necessary if the initial correspondence between the small set of reconstructed landmarks $L$ and the target m-rep is good. For majority of the testing cases, to be shown in next section, one iteration of the optimization of the objective function $F\left(\mathbf{M}_{t}^{\prime}\right)$ is sufficient. However, by updating an initial correspondence that is of poor quality, the overall algorithm is more robust to correspondence errors.

\subsection{Deformation Propagation to Substructures}

The target models before and after the registration are used to imply a deformation field for the interior and the adjacent exterior volume of the target object. The deformation field is propagated to the substructure volume, voxel by voxel. Because of the enforced legality of the deformed $\mathbf{M}^{\prime}$ by the component $F_{\text {leg }}$, the volumetric legalities of both the models, before and after the registration, are guaranteed. Therefore, the implied deformation field is guaranteed to be legal. Next section evaluates the proposed method.

\section{Result}

In order to evaluate the proposed method, a set of kidney models with synthetic deformations is generated. Synthetic data provide the ground truth to better evaluate our method. Also, the impact of reconstruction errors by the SFM method is studied. One set of in vivo data is also used to test our method. 
The rationale of using synthetic deformations is that the types of deformations a kidney undergoes during an MIS can be well described and modeled by experienced surgeons so it is a reasonable approximation to population deformations. However, our method can be applied to dynamic CT or range data sets to learn arguably more realistic organ deformations.

\subsection{Generating Synthetic Testing Data}

There are two parts of data generations:

- Generation of the statistical deformation models: 20 kidney m-reps $\mathbf{M}_{k i d, i}$, $i \in[1,20]$ from different patients are used. A series of simulated deformations are applied to each kidney m-rep. Each kidney m-rep and its deformed shapes are used to build a statistical deformation model $\Delta \mathbf{M}_{k i d, i}\left(\left\{c_{j}\right\}\right)$ of the reference m-rep $\overline{\mathbf{M}}_{k i d, i}$. Each statistical deformation model is then used to guide the registration of all the other 19 kidneys. In total there are $20 \times 19$ registration results. A tumor m-rep is also added to each kidney m-rep.

- Generation of video sequences for SFM reconstructions: a diffeomorphic deformation, independent from the deformations used to generate the statistical deformation models, is applied to the m-rep implied surface meshes of the kidney and tumor.

A kidney texture image, stitched from an in vivo video, is used as the texture for each deformed kidney mesh $\mathbf{M e s h}_{k i d, i}$. Using the parameters of a calibrated Stryker laparoscope, a series of 15 images $\mathbf{I}_{i}$ are generated at the resolution of $640 \times 480$ to cover about half of each kidney surface, assuming no deformations among these image frames. A set of 100 surface points are randomly selected as the ground truth reconstructed surface points $\mathbf{L}_{\text {truth }, a l l, i} .6$ to 9 landmarks of anatomical significance are selected from each mesh as the set $\mathbf{L}_{t r u t h, i}$. Initial correspondence between $\mathbf{L}_{t r u t h, i}$ and the $\mathrm{m}$ rep $\mathbf{M}_{i}$ is also automatically established.

\subsection{Experimental Results from Synthetic Data}

Guided by the statistical deformation model learned from the reference kidney $\mathbf{M}_{i} \in[1,20]$, each $\mathrm{m}$-rep $\mathbf{M}_{j}, j \neq i$ was registered into its video sequence $\mathbf{I}_{j}$ to acquire the registered m-rep $\mathbf{M}_{i, j}^{\prime}$. Each $\mathbf{M}_{j, i}^{\prime}$ was compared to the ground truth landmark points $\mathbf{L}_{t r u t h, j}$ to calculate the average point-to-point distance (APD), and $\mathbf{M}_{j, i}^{\prime}$ was also compared to each ground truth mesh $\mathbf{M e s h} \mathbf{h}_{k i d, j}$ to calculate the average surface distance (ASD) Each $\mathbf{M}_{j, i}^{\prime}$ was then used to estimate and apply propagation deformations to its tumor model. The deformed tumor model was compared to the ground truth tumor mesh $\mathbf{M e s h} \mathbf{h}_{\text {tumor }, j}$ to calculate the ASD. A deformation model and 3 testing kidney models are shown in figure 1.

All the experiments were conducted with different levels of Gaussian noise added to the reconstructed surface points $\mathbf{L}_{a l l, j}$ : the standard deviations are 1,3 , and 5 voxels. The size of 1 voxel is approximately $0.78 \mathrm{~mm}$. The average experimental results are shown in table 1 . The deformation propagation errors of the tumor are 


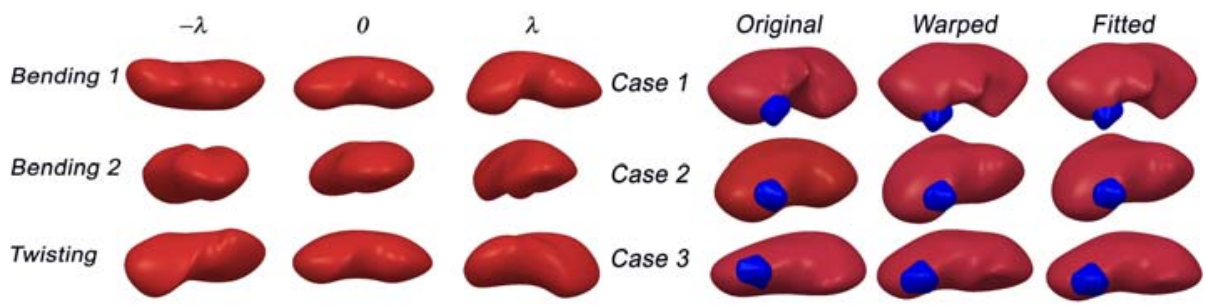

Fig. 1. Left: 3 main modes of a deformation model: each row shows one mode from $-\lambda$, 0 , to $\lambda$; Right: 3 testing kidney m-reps, from left to right: the original target kidney m-rep $\mathbf{M}_{i}$ (in red), the ground truth surface mesh of the kidney and tumor (in blue) reconstructed from warped object volume, and the registered $m$-rep $\mathbf{M}^{\prime}$ with the deformation applied to its attached tumor model

Table 1. All units are in voxels, with the size of $0.78 \mathrm{~mm}$, except the number of iterations

\begin{tabular}{|l|l|l|l|l|l|}
\hline $\begin{array}{l}\text { STD } \\
\text { of Noise }\end{array}$ & $\begin{array}{l}\text { Kidney } \\
\text { Avg. APD }\end{array}$ & $\begin{array}{l}\text { Kidney } \\
\text { Avg. ASD }\end{array}$ & $\begin{array}{l}\text { Tumor } \\
\text { Avg. ASD }\end{array}$ & $\begin{array}{l}\text { Avg. Number } \\
\text { of Iterations }\end{array}$ & $\begin{array}{l}\text { Kidney Avg. ASD } \\
\text { Without ICP }\end{array}$ \\
\hline 1 & $0.57 \pm 0.88$ & $0.75 \pm 0.65$ & $1.38 \pm 0.69$ & $1.36 \pm 0.53$ & $1.14 \pm 0.78$ \\
3 & $2.24 \pm 1.35$ & $2.59 \pm 0.95$ & $3.25 \pm 1.06$ & $3.00 \pm 0.87$ & $3.76 \pm 2.03$ \\
5 & $3.27 \pm 1.45$ & $3.41 \pm 1.30$ & $4.19 \pm 1.41$ & $5.81 \pm 1.54$ & $6.82 \pm 3.44$ \\
\hline
\end{tabular}

bigger than the registration errors of kidneys, which is expected. As the noise level for the reconstruction error increases, the registration errors increase too, but at a slower pace. At a lower noise level, most registrations only require 1 iteration of optimization. However, the ICP step is necessary to keep the registration robust as the noise increases. The last column shows that the registration results deteriorate rapidly without the ICP to correct a poor initial correspondence.

\subsection{Results from a Set of in vivo Data}

A CT scan of $1 \mathrm{~mm} \times 1 \mathrm{~mm} \times 3 \mathrm{~mm}$ was used to build the initial m-rep model for the target kidney. Because of the lack of enough training data, the deformation model built from the synthetic data was used to guide the registration of the m-rep to the video sequence. There is no ground truth surface mesh available. A dense set of 200 surface points were reconstructed from the video sequence and were used in the registration. The average distance between the surface points to the implied boundary surface of the registered m-rep is $2.65 \mathrm{~mm}$.

\section{Discussion}

Our method has the advantages as follows: the registration via deformation cloning uses a statistical deformation model learned from often very limited training data, and the registration completes the deformed target object; only 
a small number of reconstructed landmarks are required as long as a good correspondence between the landmark set and the target model is established; the registered deformations of the target object can be used to estimate deformations to important substructures.

We are working on live animal experiments to further validate our method. One challenging but rewarding extension of our method is to combine and apply multiple deformation models to a new target object.

\section{References}

1. Devernay, F., Mourgues, F., Coste-Maniere, F.: Towards endoscopic augmented reality for robotically assisted minimally invasive cardiac surgery. In: Proceedings of the International Workshop on Medical Imaging and Augmented Reality (MIAR 2001), pp. 16-20 (2001)

2. Lau, W., Ramey, N., Corso, J., Thakor, N., Hager, G.: Stereo-based endoscopic tracking of cardiac surface deformation. In: Barillot, C., Haynor, D.R., Hellier, P. (eds.) MICCAI 2004. LNCS, vol. 3217, pp. 494-501. Springer, Heidelberg (2004)

3. Lo, B.P., Scarzanella, M.V., Stoyanov, D., Yang, G.Z.: Belief propagation for depth cue fusion in minimally invasive surgery. In: Metaxas, D., Axel, L., Fichtinger, G., Székely, G. (eds.) MICCAI 2008, Part II. LNCS, vol. 5242, pp. 104-112. Springer, Heidelberg (2008)

4. Hu, M., Edwards, P., Figl, M., Hawkes, D.J.: 3D reconstruction of internal organ surfaces for minimal invasive surgery. In: Ayache, N., Ourselin, S., Maeder, A. (eds.) MICCAI 2007, Part I. LNCS, vol. 4791, pp. 68-77. Springer, Heidelberg (2007)

5. Mohamed, A., Davatzikos, C., Taylor, R.: A combined statistical and biomechanical model for estimation of intra-operative prostate deformation. In: Dohi, T., Kikinis, R. (eds.) MICCAI 2002. LNCS, vol. 2489, pp. 452-460. Springer, Heidelberg (2002)

6. Hu, Y., Morgan, D., Ahmed, H.U., Pends, D., Sahu, M., Allen, C., Emberton, M., Hawkes, D., Barratt, D.: A statistical motion model based on biomechanical simulations for data fusion during image-guided prostate interventions. In: Metaxas, D., Axel, L., Fichtinger, G., Székely, G. (eds.) MICCAI 2008, Part I. LNCS, vol. 5241, pp. 737-744. Springer, Heidelberg (2008)

7. Yuen, S.G., Kesner, S.B., Vasilyev, N.V., Nido, P.J.D., Howe, R.D.: 3D ultrasoundguided motion compensation system for beating heart mitral valve repair. In: Metaxas, D., Axel, L., Fichtinger, G., Székely, G. (eds.) MICCAI 2008, Part I. LNCS, vol. 5241, pp. 711-719. Springer, Heidelberg (2008)

8. Pizer, S.M., Fletcher, T., Fridman, Y., Fritsch, D.S., Gash, A.G., Glotzer, J.M., Joshi, S., Thall, A., Tracton, G., Yushkevich, P., Chaney, E.L.: Deformable m-reps for $3 \mathrm{~d}$ medical image segmentation. International Journal of Computer Vision Special UNC-MIDAG issue 55(2), 85-106 (2003)

9. Fletcher, P.T., Lu, C., Pizer, S.M., Joshi, S.: Principal geodesic analysis for the nonlinear study of shape. Transactions on Medical Imaging (TMI) 23(8), 995-1005 (2004)

10. Lu, C., Pizer, S.M., Joshi, S., Jeong, J.Y.: Statistical multi-object shape models. IJCV 75(3), 387-404 (2007)

11. Han, Q., Merck, D., Levy, J., Villarruel, C., Damon, J.N., Chaney, E.L., Pizer, S.M.: Geometrically proper models in statistical training. In: Karssemeijer, N., Lelieveldt, B. (eds.) IPMI 2007. LNCS, vol. 4584, pp. 751-762. Springer, Heidelberg (2007)

12. Besl, P., Mckay, N.: A method for registration of 3-d shapes. IEEE TPAMI 14(2), 239-256 (1992) 